\title{
A New Optimal Velocity Control Function Considering Driver's Anticipation Effect
}

\author{
B.G. Cao \\ The State Key Laboratory of Automobile Dynamic Simulation \\ Automobile College of Jilin University \\ Transportation College of Jilin University \\ No.5988, Renmin Street, Changchun City, Jilin Province, China
}

\begin{abstract}
Considering longitudinal spatial deviations from expected referenced equilibrium point (plan), a new two dimensional (2-d) optimal velocity function considering driver's nonlinear adjustment effect is presented. We call it as super desired velocity, simplified as SDV. Unlike existing optimal velocity (OV) which is based on the absolute headway, SDV is dependent on referenced equilibrium headway, namely based on the relative headway. They are composed of two parts: desired velocity term and nonlinear adjustment term to reach the desired velocity value. The performance of new optimal velocity model is illustrated in detail by numerical simulation methods. The numerical simulation results show that SDV can model driver's nonlinear anticipation better than the existing models.
\end{abstract}

Keywords- optimal velocity; driver anticipation; referenced point; equilibrium

\section{INTRODUCTION}

An increasing number of traffic flow models have been developed to study various complex traffic phenomena [1-12]. However, these models cannot describe various complex phenomena resulted by driver anticipation since they did not consider this factor. In real traffic system, all drivers may not hope stop-and-go traffic phenomenon. They more hope a steady driving state. According to Lighthill and Whitham (1955) and Richards (1956)--LWR theory [4], when traffic flow is in the equilibrium state, all successive cars run by the same velocity (referenced equilibrium velocity, simplified as $v_{r}$ ), and have the same distance headway (referenced equilibrium headway $h_{r}$ ). Therefore, in order to maintain the steady driving state, when

(1) $\left(h>h_{r}\right)$, in order to keep up with the equilibrium state, the driver of following car will expect a bigger velocity than the leading car to compensate the positive deviation from equilibrium position dependent on the velocity of the leading car.

(2) $\left(h<h_{r}\right)$, similarly, in order to keep up with the equilibrium state, the driver of following car expects a smaller velocity than the leading car to compensate the negative distance deviation.

(3) $\left(h=h_{r}\right)$, the driver of following car expects to choose the velocity of the leading one to be the desired velocity.
Definitely, from the viewpoint of deviation from dynamic equilibrium headway dependent on the equilibrium velocity. Following cars can be generalized into the following three traffic states in Fig.1.

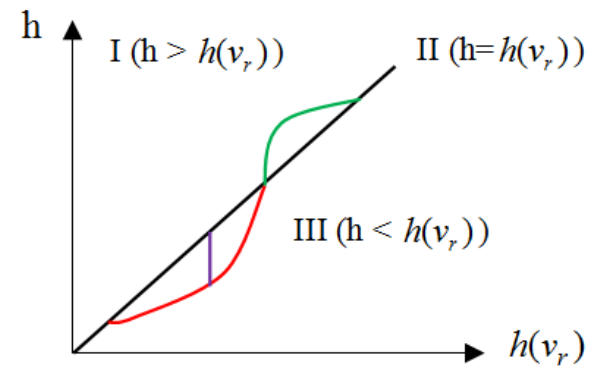

FIGURE I. TRAFFIC STATES BASED REFERENCED EQUILIBRIUM HEADWAY.

In Fig. $1, h$ is the distance headway (relative distance to the leading car) as described in earlier models, $h\left(v_{r}\right)$ is a new variable called as referenced equilibrium distance headway (legal safe headway). Referenced equilibrium headway $h_{r}$ and referenced equilibrium velocity $v_{r}$ are dependent, and they satisfy the mathematical equation $h_{r}=h\left(v_{r}\right)$. The referenced equilibrium headway $h\left(v_{r}\right)$ divides the headway $h$ into three regions I, II, III in Fig. 1. Naturally, the deviation value $\left(h_{n}-h\left(v_{r}\right)\right)$ from referenced equilibrium distance $h\left(v_{r}\right)$ comes as the motive force of the driver's nonlinear anticipation term composed of an extended hyperbolic function which will be described in detail in section II.

\section{THE FORMATION OF SVD}

Based on above discussion, a new optimal velocity function (SDV) is proposed as the mathematical equation (1):

$$
\mathrm{V}_{\mathrm{d}}\left(h_{n}, v_{r}\right)=v_{r}+C_{1}\left[\tanh C_{2}\left(h_{n}-h\left(v_{r}\right)\right)\right],
$$

where, $h_{n}, v_{r}$ are independent variables. $h, v_{r}$ are dependent variables.

As be shown in Eq. (1), we can see that $\mathrm{V}_{\mathrm{d}}\left(h_{n}, v_{r}\right)$ includes two parts, one is the referenced equilibrium velocity (equilibrium term), another is a hyperbolic term composed of 
the deviation magnitude of distance headway from referenced equilibrium position $h\left(v_{r}\right)$ we call anticipated term. $C_{1}$, $C_{2}$ are coefficients to be calibrated.

The new $\mathrm{V}_{\mathrm{d}}\left(h_{n}, v_{r}\right)$ has the following characteristics:

$\mathrm{V}_{\mathrm{d}}\left(h_{n}, v_{r}\right)$ is a set of expecting velocity curves dependent on referenced velocity $v_{r}$, there is an expected velocity curve (function of $h_{n}$ ) corresponding to every referenced velocity $v_{r}$. For every expected velocity curve, there is a point of inflection at $v_{r}$. All points of inflection link into a line which corresponds to equilibrium headway-velocity relationship.

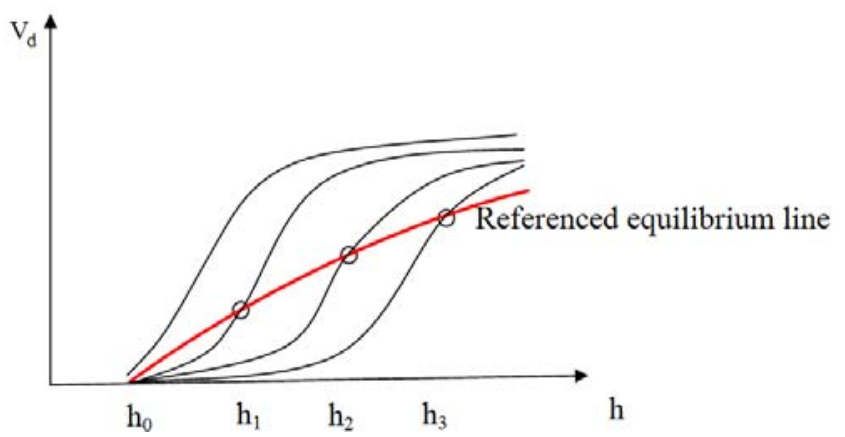

FIGURE II. TRAFFIC STATE REGIONS BASED REFERENCED EQUILIBRIUM HEADWAY.

However, in the existing OV model [5, 6] and GF model [7], the optimal velocity is only dependent on single variable: distance headway to the leading car. They did not consider the effects of the velocity and driver anticipation on the following car.

Further research shows that the acceleration and deceleration response of driver and vehicle are different. So, another improvement is that the asymmetry for accelerating and decelerating are considered in the new desired velocity model.

So we give different thresholds for $C_{1}$.

When $h_{n}>h\left(v_{r}\right)$,

$$
\begin{array}{r}
\mathrm{V}_{\mathrm{d}}\left(h_{n}, v_{r}\right)=v_{r}+C_{1}\left[\tanh C_{2}\left(h_{n}-h\left(v_{r}\right)\right)\right], \\
C_{1} \in\left[0,\left(v_{m}-v_{r}\right)\right] . \quad(2)
\end{array}
$$

When $h_{n}<h_{r}\left(v_{r}\right)$,

$\mathrm{V}_{\mathrm{d}}\left(h_{n}, v_{r}\right)=v_{r}+C_{1}\left[\tanh C_{2}\left(h_{n}-h_{r}\left(v_{r}\right)\right)\right], C_{1} \in\left[0, v_{r}\right]$.

When $h_{n}=h_{r}\left(v_{r}\right)$,

$$
\mathrm{V}_{\mathrm{d}}\left(h_{n}, v_{r}\right)=v_{r} .
$$

Thus, the value of $\mathrm{V}_{\mathrm{d}}\left(h_{n}, v_{r}\right)$ is all the time in [0, $\left.v_{\max }\right]$, and without producing the unpractical desired velocity.
We can see that $\mathrm{V}_{\mathrm{d}}\left(h_{n}, v_{r}\right)$ has upper and lower bounds, so smaller or bigger speeds are avoided in preceding models.

And with the following properties:

(1) $\mathrm{V}_{\mathrm{d}}\left(h_{n}, v_{r}\right)$ is a monotonically increasing function

(2) $\mathrm{V}_{\mathrm{d}}\left(h_{n}, v_{r}\right)$ can not be infinite, which has an upper bound $v_{\max }$, which depends on the vehicle dynamic performance.

\section{NUMERICAL SIMULATION AND COMPARISON ANALYSIS}

In the following, we will give the numerical simulation comparison of SDV model proposed in this paper with existing OV and GF models.

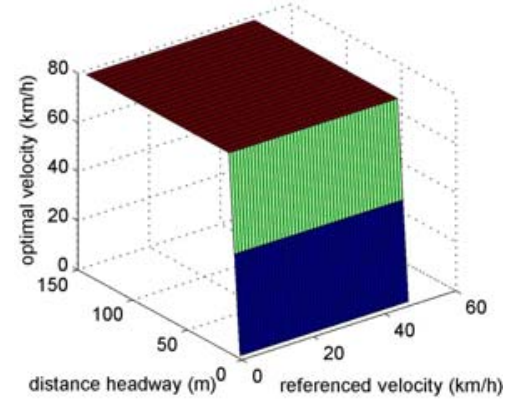

(a)

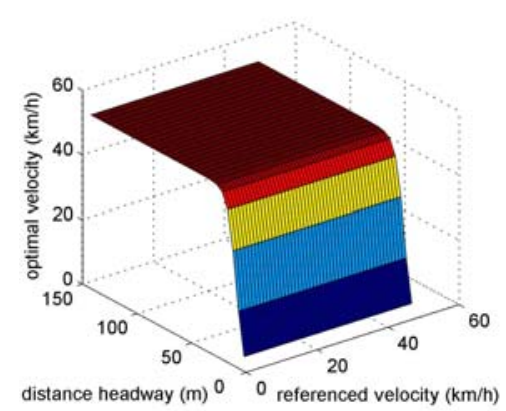

(b)

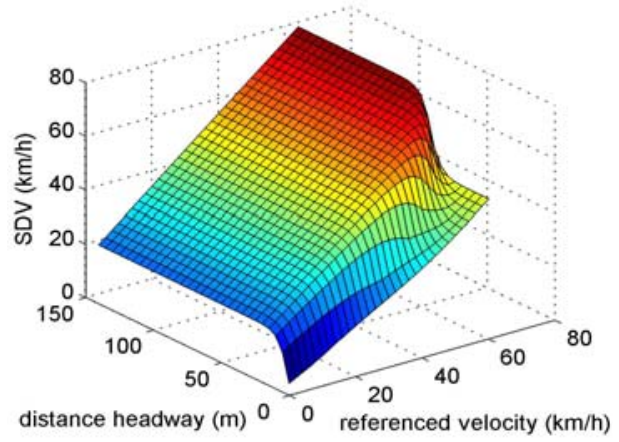

(c)

FIGURE III. OV, GF AND SDV NUMERICAL SIMULATION RESULTS. 
In Fig. 3(a) and 3(b), we can see that the optimal velocity OV and GF depend on distance headway solely, irrelevant to referenced velocity. In Fig. 3(c), we can see that the new SDV model is not only related to distance headway, but also the referenced velocity.

As described in the above Fig. 3(c), we can see that some anticipated velocities exist above equilibrium plan or reference plan, some exist below the equilibrium plan according to the relationship of distance headway and referenced equilibrium velocity.

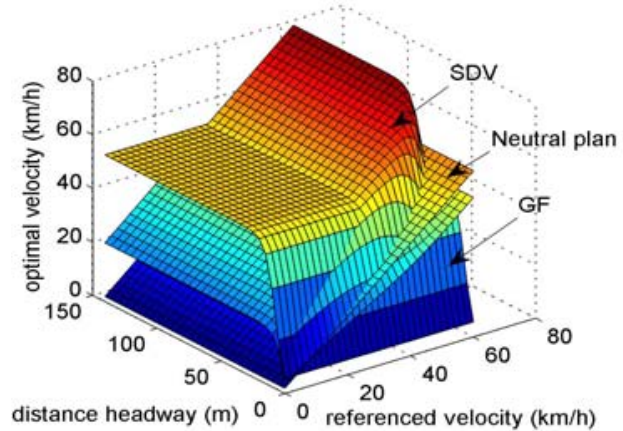

FIGURE IV. NUMERICAL SIMULATION COMPARISON OF SDV AND GF.

To illustrate the improvement in detail, a comparison of SDV and GF numerical simulation results is given in Fig. 4, we can see SDV model can describe driver nonlinear anticipated effect well with a comparison with GF model. The model parameters are taken with $C_{1}=20 \mathrm{~m} / \mathrm{s}$ for positive deviation from equilibrium headway and $C_{1}=10 \mathrm{~m} / \mathrm{s}$ for negative deviation respectively. $C_{2}=0.13$.

As can be seen in Fig. 4, according to the relationship of distance headway $h_{n}$ and referenced equilibrium headway $h\left(v_{r}\right)$, when traffic flow is in the equilibrium state, there exist $h_{n}=h_{r}\left(v_{r}\right), \mathrm{V}_{\mathrm{d}}\left(h_{n}, v_{r}\right)=v_{e}$, so the new desired velocity function surface becomes a 2-dimension flat surface that we can call as referenced equilibrium plan (neutral plan). In the referenced neutral plan, all cars are in the traffic flow equilibrium state, namely, all distance headways are identical to equilibrium headway and all velocities identical to equilibrium velocity.

\section{CONCLUSIONS AND PROSPECT}

A new dynamic 2-dimension reference points (plan) for driver decision-making based on the equilibrium velocity and equilibrium headway is put forward in this paper. Further, a new 2-D desired velocity model is proposed based on dynamic equilibrium referenced points (plan), which includes two parts, one is the referenced equilibrium velocity, another is an anticipation adjustment term to reach the referenced equilibrium velocity and the anticipation adjustment term is composed of an extended hyperbolic term to model driver nonlinear spatial anticipated effect. Finally, the asymmetry reaction is considered in SDV model considering positive deviation and negative deviation from referenced points (plan).

In fact, considering the complexity of real traffic system, new optimal velocity model proposed in this paper still need a larger number of experimental data to further test its application performance, and there is still a lot of work to be further studied and improved, such as differences between drivers, driver cognition ability, driving urgent degree, road conditions etc..

\section{ACKNOWLEDGMENT}

This work undertaken in this paper was supported by National Natural Science Foundation of China (No. 61104168) and China Postdoctoral Science Foundation (No. 20110491306).

\section{REFERENCES}

[1] L.A. Pipes, An Operational Analysis of Traffic Dynamics. Journal of Applied Physics, 24, pp. 274-281, 1953.

[2] F. E. Chandler, R. Herman, E. W. Montroll, Traffic Dynamics: Studies in Car Following. Operations Research, 6, pp. 165-184, 1958.

[3] D. L. Gerlough, M. J. Huber, Traffic Flow Theory. National Research Council, Washington D. C., 1975.

[4] D. May Adolf, Traffic Flow Fundamentals. Prentice Hall, Inc., New Jersey, 1990.

[5] M. Bando, K. Hasebe, Dynamic model of traffic congestion and numerical simulation. Physical Review E, 51, pp. 1035-1042, 1995.

[6] M. Bando, K. Hasebe, Analysis of optimal velocity model with explicit delay. Physical Review E, 58, pp. 5429-5435, 1998.

[7] D. Helbing, B. Tilch, Generalized force model of traffic dynamics. Physical Review E, 58, pp. 133-138, 1998.

[8] R. Jiang, Q.S. Wu, Z.J. Zhu, Full velocity difference model for carfollowing theory. Physical Review E, 64, 017101, 2001.

[9] R. Jiang, Q.S. Wu, Z.J. Zhu, A new continuum model for traffic flow and numerical tests. Transportation Research Part B, 36, pp. 405-419, 2002.

[10] X.W. Tang, X.S. Zhang, K.Q. Lu, Traffic flow and Granular Flow. Zhejiang University Press, 2004. (in Chinese)

[11] H.M. Zhang, A non-equilibrium traffic model devoid of gas-like behaviour. Transportation Research Part B, 36, pp. 275-290, 2002.

[12] J.A. Laval, Hysteresis in traffic flow revisited: an improved measurement method. Transportation Research Part B, 36, pp. 385-391, 2011. 